\title{
LARGE SCALE MINIMAX OPTIMIZATION OF MICROWAVE MULTIPLEXERS
}

\author{
J.W. Bandler*†, S.H. Chen*, S. Daijavad*, W. Kellermann*, M. Renault* and Q.J. Zhang*
}

\begin{abstract}
A new technique for contiguous-band multiplexer design involving an arbitrarily large number of channels and design parameters is described. The technique, based on network decomposition and sensitivity measures of practical microwave multiplexer structures, employs a sequence of relatively small optimization problems which correspond to growing a multiplexer by adding one or more channels at a time to the structure. The approach is illustrated by a 16channel, $12 \mathrm{GHz}$ multiplexer design involving 240 nonlinear design variables.
\end{abstract}

\section{INTRODUCTION}

Progress in the area of multiplexer design and tuning has been substantially hindered by the dimensionality of the problems that have to be solved to provide the designer with parameter values ensuring the desired performance. Recent developments in computer-aided design techniques led to the utilization of optimization methods to determine the optimal multiplexer parameters [1-2]. Practical multiplexer designs reported in the literature involve up to 12 channels [3-4].

If all possible parameters of a 12-channel system are to be optimized, i.e., waveguide spacings, input-output and filter couplings, we have an optimization problem with 180 nonlinear optimization variables (assuming 6th order filters). The number of functions, depending on the types of responses to be optimized, is usually about 100-300 (corresponding to a discrete set of sample points adequately approximating the continuous response). Our computational experience with this type of problem indicates that memory requirements to solve such a large design problem can exceed the capability of such powerful systems as the CDC Cyber 170/730 if mass storage devices are not to be used.

To overcome the dimensionality problem we formulate the design of a contiguous-band multiplexer structure with an arbitrarily large number of channels and design parameters as a sequence of appropriately defined smaller optimization problems.

The approach proposed in this paper is based on network decomposition and sensitivity measures for multiplexer structures. This type of theoretical analysis verifies that in the multiplexer structure there exists a natural decoupling of the system responses with respect to groups of parameters. By exploiting this feature of multiplexer structures we suggest a method of growing a multiplexer by adding sequentially one channel or a few channels at a time to the overall structure. Each new addition to the multiplexer is optimally integrated into the existing structure to ensure that the overall structure satisfies the design specifications.

The approach has two very important consequences. First, it allows us to design effectively multiplexers of arbitrary size using a small or medium size computer. Second, it creates the basis for successful postproduction tuning algorithms for multiplexer realizations.

\section{DESCRIPTION OF THE APPROACH}

Suppose we want to design an $\mathrm{N}$-channel waveguide manifold multiplexer satisfying given specifications on the common port return loss and individual channel insertion loss functions. To achieve that goal we could perform a sequence of $\mathrm{N}-1$ optimizations. In each of

* Simulation Optimization Systems Research Laboratory, Department of Electrical and Computer Engineering, McMaster University, Hamilton, Canada L8S 4L7.

$\dagger$ President, Optimization Systems Associates, 163 Watson's Lane, Dundas, Ontario, Canada, L9H 6L1. 
those optimizations we add a new channel to the existing $\mathrm{k}$-channel multiplexer. The resulting $(k+1)$-channel structure is then optimized with optimization variables taken from channels $k$ and $k+1$ and specifications imposed on responses in channels $k-1, k$ and $k+1$. Sample frequencies are selected from the frequency range covering channels $k-1, k$ and $k+1$. Formally, let $\mathbf{x}_{\mathrm{k}}, \mathrm{k}=1,2, \ldots, \mathrm{N}$, be the set of all design parameters associated with the $\mathrm{kth}$ channel and let $\Omega_{\mathrm{k}} \triangleq\left[\omega_{\mathrm{L}}^{\mathrm{k}}, \omega_{\mathrm{U}^{\mathrm{k}}}\right]$ be the frequency interval related to the $\mathrm{kth}$ channel. The $\mathrm{kth}$ optimization problem, $\mathrm{k}=1, \ldots, \mathrm{N}-1$, corresponds to the addition of the $(\mathrm{k}+1)$ th channel. It is defined as

$$
\overline{\min }_{\overline{\mathbf{x}}_{\mathrm{k}}} \max _{\mathrm{j} \in \mathrm{J}_{\mathrm{k}}} \mathbf{f}_{\mathrm{j}}(\mathbf{x})
$$

where $\overline{\mathbf{x}}_{\mathrm{k}} \triangleq\left[\mathbf{x}_{\mathrm{k}}^{\mathrm{T}} \mathbf{x}_{\mathrm{k}+1}{ }^{\mathrm{T}}\right]^{\mathrm{T}}, \mathbf{x} \triangleq\left[\mathbf{x}_{1}^{\mathrm{T}} \mathbf{x}_{2}^{\mathrm{T}} \ldots \mathbf{x}_{\mathrm{k}+1}{ }^{\mathrm{T}}\right]^{\mathrm{T}}$ and $\mathrm{J}_{\mathrm{k}}$ is the index set for minimax functions for the kth subproblem.

The error functions $f_{j}(x), j \in J_{k}$, are of the form

$$
\begin{aligned}
& w_{U p}^{1}\left(\omega_{i}\right)\left(F_{p}^{1}\left(x, \omega_{i}\right)-S_{U p}^{1}\left(\omega_{i}\right)\right) \\
& -w_{L p}^{1}\left(\omega_{i}\right)\left(F_{p}^{1}\left(x, \omega_{i}\right)-S_{L p}^{1}\left(\omega_{i}\right)\right) \\
& w_{U}^{2}\left(\omega_{i}\right)\left(F^{2}\left(x, \omega_{i}\right)-S_{U}^{2}\left(\omega_{i}\right)\right) \\
& -w_{L}^{2}\left(\omega_{i}\right)\left(F^{2}\left(x, \omega_{i}\right)-S_{L}^{2}\left(\omega_{i}\right)\right)
\end{aligned}
$$

where $\omega_{\mathrm{i}} \in \bar{\Omega}_{\mathrm{k}} \triangleq \Omega_{\mathrm{k}+1} \cup \Omega_{\mathrm{k}} \cup \Omega_{\mathrm{k}-1}, \Omega_{0} \triangleq \varnothing$ ( $\varnothing$ denotes an empty set), $\mathrm{p} \in\{\mathrm{k}+1, \mathrm{k}, \mathrm{k}-1\}$, $\mathrm{F}_{\mathrm{p}} 1\left(\mathrm{x}, \omega_{\mathrm{i}}\right)$ is the insertion loss for the pth channel at the ith frequency, $\mathrm{F}^{2}\left(\mathrm{x}, \omega_{\mathrm{i}}\right)$ is the return loss at the common port at the ith frequency, $S_{U p} 1\left(\omega_{i}\right)\left(S_{L_{p}} 1\left(\omega_{i}\right)\right)$ is the upper (lower) specification on insertion loss of the pth channel at the ith frequency, $S_{U^{2}}{ }^{2}\left(\omega_{i}\right)\left(S_{L}{ }^{2}\left(\omega_{i}\right)\right)$ is the upper (lower) specification on return loss at the ith frequency, and $w_{U_{p}}{ }^{1}, w_{L p}{ }^{1}, w_{U^{2}}, w_{L}{ }^{2}$ are the arbitrary userchosen nonnegative weighting factors.

It is desirable to have good starting values for the optimization problems described in (1). We suggest the following procedure before the kth subproblem is actually solved. For contiguousband multiplexers the starting values for parameters in the $(k+1)$ th channel may be obtained by duplicating the corresponding parameters for channel $k$ except for the spacing. The starting value for the $(k+1)$ th spacing (distance from the short circuit) is calculated using the following formula

$$
\ell_{k+1}=\ell_{k} \frac{(k+1) \Lambda_{g_{k+1}}}{k \lambda_{g_{k}}}
$$

where $\lambda_{g_{k}}$ is the guide wavelength at center frequency $\omega_{0_{k}}$. Similarly, the design parameters of channel $\mathrm{k}$ are replaced by the corresponding values for channel $(\mathrm{k}-1)$ with the spacing correction given in (6) with appropriate indices.

To preserve good performance of the channels already optimized we relax the specification on the channel being added, keeping in mind that the new addition will be optimized with respect to the original specification in the $(\mathrm{k}+1)$ th optimization.

To solve the subproblem defined in (1) we use a recently developed minimax algorithm [2]. The algorithm requires functions and their first-order derivatives to be supplied. An efficient approach to the simulation of multiplexer responses and their first-order sensitivities with respect to all network parameters and frequency has been recently described [5].

Due to the approximations made in this approach (weak couplings are neglected) the performance of some of the channels that are not controlled may deteriorate as the process progresses. This deterioration, however, is not serious and can be readily corrected by reoptimizing those channels whose performance has deteriorated.

An important feature of our new approach to multiplexer design is an efficient representation of the constant parts of the multiplexer during the solution process of the subproblem defined in (1). 
It has been observed that parameters associated with a particular channel of the multiplexer structure have a strong effect on responses corresponding to that channel and a weak effect on responses related to other channels. To describe this phenomenon we construct an $\mathrm{N} \times \mathrm{N}$ sensitivity matrix $\mathbf{A}$ whose $(q, k)$ th element is defined as:

where

$$
A_{q k}=\sum_{r=1}^{R} \sum_{m=1}^{M} \sum_{\omega_{i} \in \Omega_{k}} s^{T} s, q, k=1,2, \cdots, N
$$

$$
\mathbf{s}=\operatorname{diag}\left\{\mathbf{x}_{\mathrm{q}}^{\mathrm{w}}\right\} \frac{\partial \mathbf{F}^{\mathrm{r}}\left(\mathbf{x}^{\mathrm{m}}, \omega_{\mathrm{i}}\right)}{\partial \mathbf{x}_{\mathrm{q}}}
$$

is a gradient vector scaled by a predetermined vector $\mathbf{x}_{\mathrm{q}}{ }^{\mathrm{w}}$. Index $\mathbf{r}$ signifies the type of response used for sensitivity calculations, e.g., $F^{1}$ denotes insertion loss, $F^{2}$ denotes return loss, etc.

From the definition (7) the element $A_{\mathrm{qk}}$ of the sensitivity matrix $A$ represents the collective influence of variables in channel $q$ on responses associated with channel $k$. The matrix A reflects the overall correlation pattern between groups of parameters and responses of various channels. The sensitivity matrix can be further normalized and made sparse for convenient reference.

Tables I and II show sensitivity matrices obtained by calculating sensitivities at 30 points (denoted $x^{m}$ in (8)) selected randomly within $\pm 40 \%$ region of the optimal point for a 16-channel multiplexer. For each element $A_{q k}$ seven frequency points were used, three of which were in the passband of the kth channel and the remaining points were in the stop-band of the kth channel. For example, for channel 1 we used the following set of frequencies (in $\mathrm{MHz}$ ):

$\{12140,12159,12161,12180,12199,12201,12220\} \subset \Omega_{1}$.

The particular response used in Table I was the common port return loss and the vector of variables $\mathbf{x}_{\mathrm{q}}$ contained all coupling parameters as well as input and output transformer ratios. The results in Table II were obtained with vector $\mathbf{x}_{\mathrm{q}}$ containing the distance from the short circuit termination of the waveguide manifold for channel $q$. The matrices have been normalized such that each element of $\mathbf{A}$ is divided by the average value of the corresponding row before normalization. The matrices are then made sparse by rounding off all entries to integers.

The A matrix in Table I is a band matrix with the bandwidth parameter equal to three, which verifies the validity of our decomposition approach. For example, growing a 4-channel multiplexer into a 5-channel multiplexer, namely, adding channel 5 , the sensitivity matrix indicates that channels 4 and 5 will both be affected. The influence on channel 4 is relatively small and can be effectively corrected by parameters in channel 4 , which in turn, affect channel 3 . Therefore specifications are imposed on channels 3,4 and 5 when parameters in channels 4 and 5 are allowed to vary.

The A matrix in Table II is close to a band matrix with dominant diagonal elements. The nonzeros in the far off-diagonal regions indicate the necessity to perform reoptimization to compensate for the deterioration of the performance of some channels due to decomposition.

\section{NUMERICAL EXAMPLE: 16-CHANNEL 12 GHz MULTIPLEXER}

We illustrate the approach described by designing a 16-channel multiplexer starting with a 12-channel optimal design presented in [5]. The structure under consideration consists of asynchronously tuned 6 th order multi-cavity filters distributed along a waveguide manifold. All design parameters, e.g., waveguide spacings (section lengths), input-output and filter coupling parameters, are directly optimized. Non-ideal effects such as junction susceptances, dissipation and dispersive effects are taken into account.

The responses of the multiplexer after adding channel 13 without optimizing it are shown in Fig. 1. Fig. 2 shows the 13-channel multiplexer optimized according to the rules described earlier, i.e., using only variables in channels 13 and 12 with specifications on responses in channels 13,12 and 11. By repeating that process three times we reach the optimal design of a 16-channel multiplexer with responses shown in Fig. 3. 


\section{CONCLUSION}

A new and powerful decomposition approach to contiguous-band multiplexer design is proposed. It employs a sequence of appropriately defined smaller optimization problems that allows the design of multiplexers with an arbitrarily large number of channels and design variables on a small computer. The validity of the approach has been demonstrated by designing a 240 variable 16-channel multiplexer. An important feature of the technique is the relevance of the sequential nature of the process to postproduction multiplexer tuning. The approach is readily extended to noncontiguous-band problems.

To the best of our knowledge, this work marks the first formal and efficient exploitation for optimization purposes of observed phenomena in multiplexer structures. We feel that this approach will have a significant impact on the future developments in computer-aided design software for microwave multiplexers and other branched structures involving several hundred design variables.

\section{REFERENCES}

[1] R.G. Egri, A.E. Williams and A.E. Atia, "A contiguous-band multiplexer design", $\underline{1983}$ IEEE Int. Microwave Symp. Digest (Boston, 1983), pp. 86-88.

[2] J.W. Bandler, W. Kellermann and K. Madsen, "A superlinearly convergent minimax algorithm for microwave circuit design", [EEE Trans. Microwave Theory Tech., vol. MTT33,1985 , pp. 1519-1530.

[3] M.H. Chen, "A 12-channel contiguous-band multiplexer at KU-band", 1983 IEEE Int. Microwave Symp. Digest (Boston, 1983), pp. 77-79.

[4] R. Tong and D. Smith, "A 12-channel contiguous-band multiplexer for satellite application", 1984 IEEE Int. Microwave Symp. Digest (San Francisco, 1984), pp. 297-298.

J.W. Bandler, S. Daijavad and Q.J. Zhang, "Exact simulation and sensitivity analysis of multiplexing networks", IEEE Trans. Microwave Theory Tech., vol. MTT-34, 1986, pp. 93-102.

\section{TABLE I}

SENSITIVITY MATRIX FOR A 16 CHANNEL MULTIPLEXER, WHERE VECTOR $\mathbf{x}_{\mathbf{q}}$ CONTAINS COUPLING PARAMETERS AND TRANSFORMER RATIOS

channels (responses)

$\begin{array}{llllllllllllllll}1 & 2 & 3 & 4 & 5 & 6 & 7 & 8 & 9 & 10 & 11 & 12 & 13 & 14 & 15 & 16\end{array}$

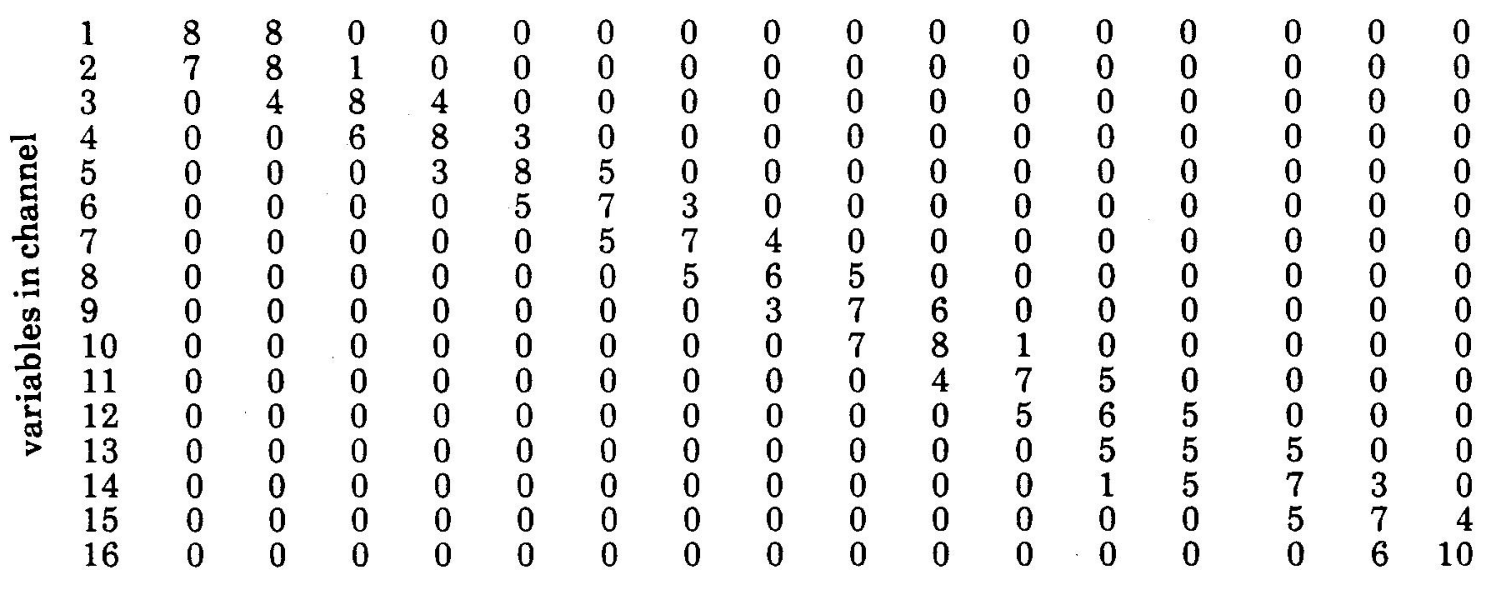


TABLE II

SENSITIVITY MATRIX FOR A 16 CHANNEL MULTIPLEXER, WHERE VECTOR $\mathbf{x}_{\mathbf{q}}$ CONTAINS THE DISTANCE FROM THE SHORT CIRCUIT

channels (responses)

$\begin{array}{llllllllllllllll}1 & 2 & 3 & 4 & 5 & 6 & 7 & 8 & 9 & 10 & 11 & 12 & 13 & 14 & 15 & 16\end{array}$

\begin{tabular}{|c|c|c|c|c|c|c|c|c|c|c|c|c|}
\hline 1 & 8 & 8 & 0 & 0 & 0 & 0 & 0 & 0 & 0 & 0 & 0 & 0 \\
\hline 2 & 7 & 8 & 0 & 0 & 0 & 0 & 0 & 0 & 0 & 0 & 0 & 0 \\
\hline 3 & 1 & 2 & 3 & 2 & 0 & 0 & 0 & 0 & 0 & 1 & 1 & 2 \\
\hline 4 & 0 & 0 & 4 & 6 & 2 & 0 & 0 & 0 & 0 & 1 & 1 & 1 \\
\hline$\Phi$ & 0 & 0 & 0 & 4 & 6 & 3 & 0 & 0 & 0 & 1 & 1 & 0 \\
\hline క్ 6 & 0 & 0 & 0 & 0 & 5 & 7 & 2 & 0 & 0 & 1 & 1 & 0 \\
\hline 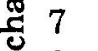 & 0 & 0 & 0 & 0 & 0 & 4 & 6 & 2 & 0 & 2 & 1 & 4 \\
\hline 88 & 0 & 0 & 0 & 0 & 0 & 1 & 3 & 5 & 3 & 2 & 2 & 0 \\
\hline 9 & 0 & 0 & 0 & 0 & 0 & 0 & 0 & 3 & 4 & 4 & 1 & 0 \\
\hline 10 & 0 & 0 & 0 & 0 & 0 & 0 & 0 & 0 & 4 & 7 & 3 & 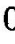 \\
\hline 11 & 0 & 0 & 0 & 0 & 0 & 0 & 0 & 0 & 0 & 1 & 3 & 5 \\
\hline 12 & 0 & 0 & 0 & 0 & 0 & 0 & 0 & 0 & 0 & 0 & 3 & 6 \\
\hline 13 & 0 & 0 & 0 & 0 & 0 & 0 & 0 & 0 & 0 & 0 & 0 & 5 \\
\hline 14 & 0 & 0 & 0 & 0 & 0 & 0 & 0 & 0 & 0 & 0 & 0 & 2 \\
\hline 15 & 0 & 0 & 0 & 0 & 0 & 0 & 0 & 0 & 0 & 0 & 0 & $c$ \\
\hline 16 & 0 & 0 & 0 & 0 & 0 & 0 & 0 & 0 & 0 & 0 & 0 & \\
\hline
\end{tabular}

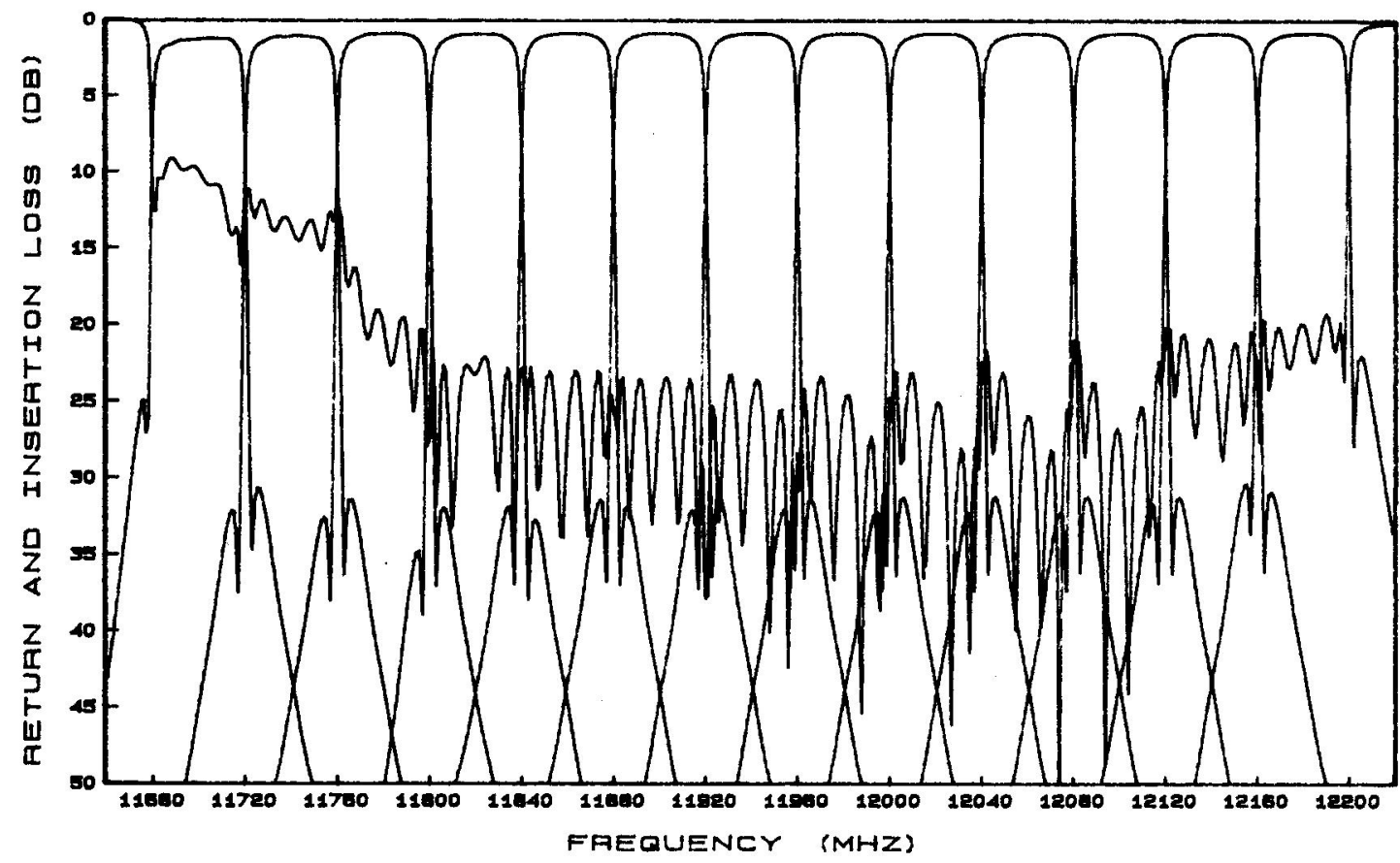

Fig. 1 Responses of nonideal 12-channel multiplexer with optimized spacings, inputoutput transformer ratios, cavity resonances and coupling parameters after adding channel 13 without optimizing it. 


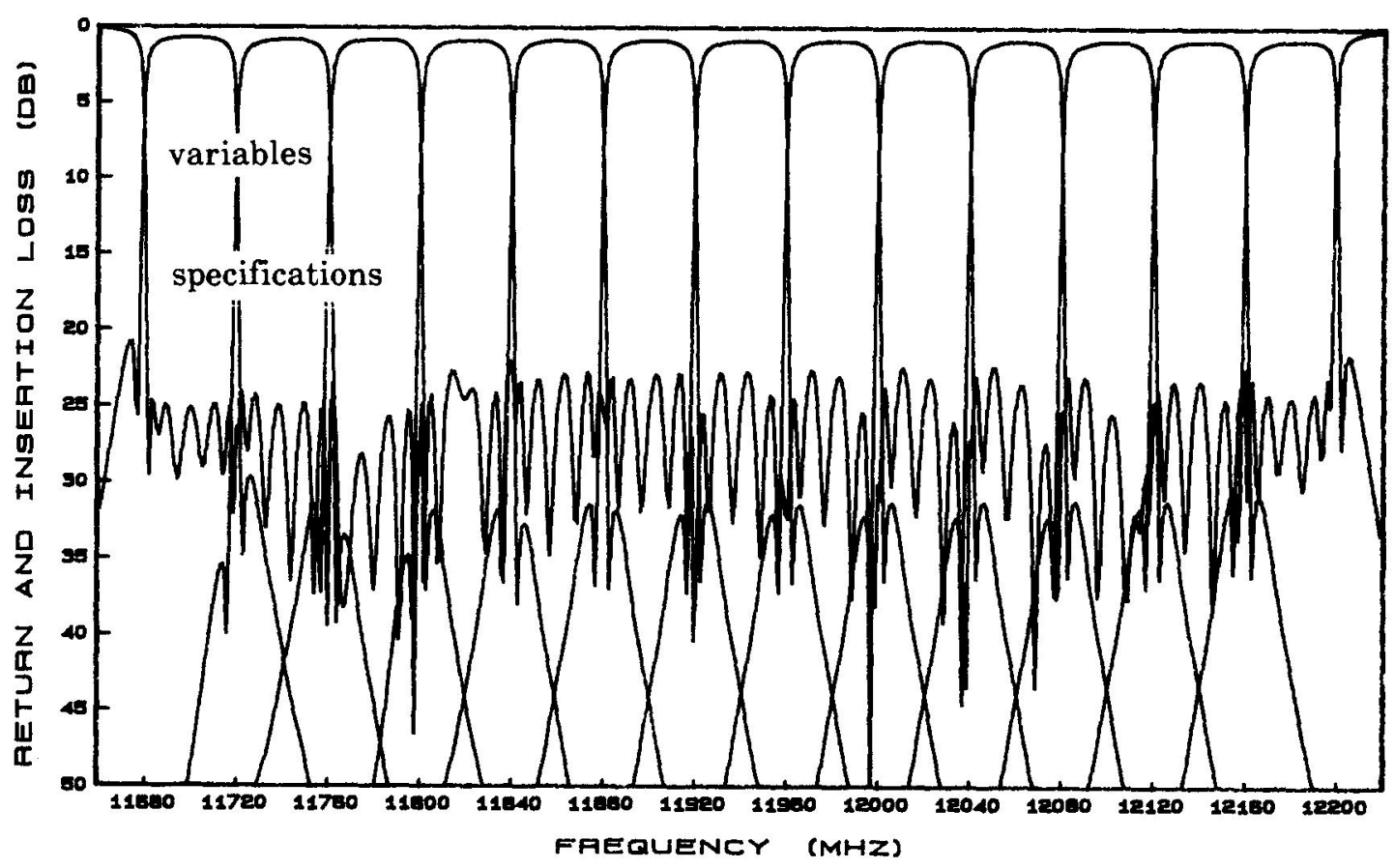

Fig. 2 Responses of the optimized nonideal 13-channel multiplexer. Optimization variables are in channels 13 and 12 , specifications are imposed on responses in channels 13,12 and 11 .

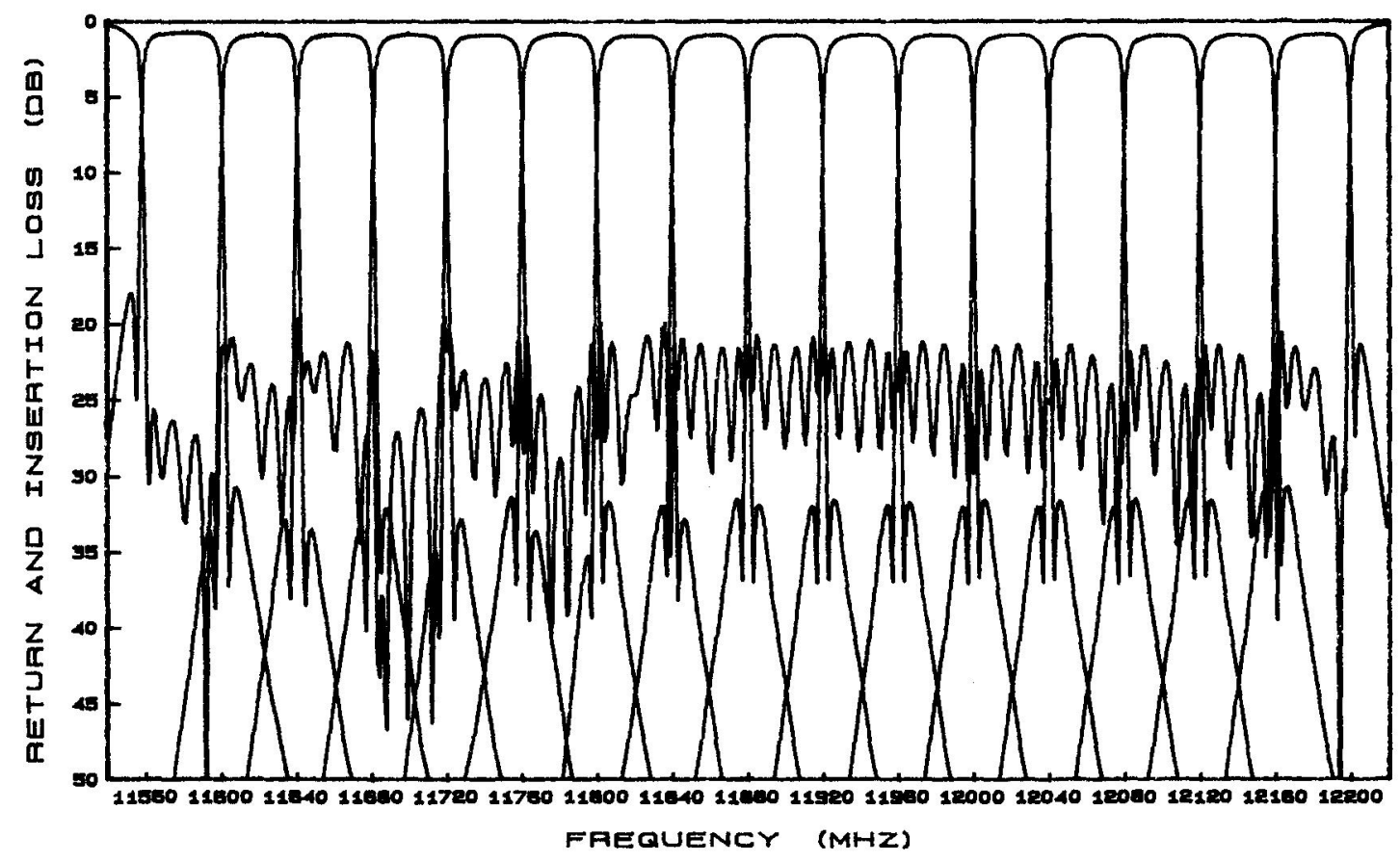

Fig. 3 Responses of a nonideal 16-channel multiplexer obtained from the optimal 12channel structure by growing one channel at a time. 